\section{- High-T's Advance}

Metal-sheathed high-temperature superconducting tapes for high-field applications [see EN 22 (1991) 157] took a major step forward with announcements this year of increases in critical current densities $J_{c}$ at $77 \mathrm{~K}$ and zero magnetic field of about $50 \%$. The work concerns Ag-clad tapes of the Bisubstituted (2223) oxide made by hot rolling, which is essentially the only viable industrial process for achieving the necessary texturing in long lengths. A Geneva University group also reported that a $10 \mathrm{~m}$ long tape achieved a record $48000 \mathrm{~A} / \mathrm{cm}^{2}$ at $4.2 \mathrm{~K}$ and $20 \mathrm{~T}$ [Physica C 217 (1993) 375] as compared to $20000 \mathrm{~A} / \mathrm{cm}^{2}$ for $\mathrm{Nb}_{3} \mathrm{Sn}$. High- $\mathrm{T}_{\mathrm{c}}$ 's can thus be envisaged for winding coils producing the highest fields provided the tapes can be adequately reinforced $\left(\mathrm{Nb}_{3} \mathrm{Sn}\right.$ conductors of the type used by Sumitomo to generate a recently announced record $21.5 \mathrm{~T}$ at $4.2 \mathrm{~K}$ are reinforced), produced in long lengths, and limitations to the packing density of the high- $T_{c}$ can be allowed for. R. Fluckiger who heads the Geneva group speaks of considerable interest in developing cryo-cooled high- $T_{c}$ magnets operating at $20 \mathrm{~K}$ without a cryogen, and in seeing if hot rolling gives high $J_{c}$ 's for the isotropic Tl-substituted compound.

\section{- 11-Year Plan for CERN}

C. Llewellyn Smith, CERN's incoming Director-General, has outlined the proposals that will be made to the CERN Council on December 17 for the proposed LHC collider. The cost is certainly above 2000 MSFR and a 11 year plan to 2005 for "somewhat in excess" of 10000 MSFR will be submitted. This is more than can perhaps be expected

\title{
One of the First to Move
}

The European Mobility Scheme for Physics Students to exchange students between participating institutions (138 at the latest count) set off this autumn with around 100 students planning to move. Katarzyna Grzegorczyk was among the first. She was about to start her fourth year at Sla saski University in Katowice, Poland, but decided instead to profit from the scheme (thanks in part by a mobility grant organized by EPS) to take courses in

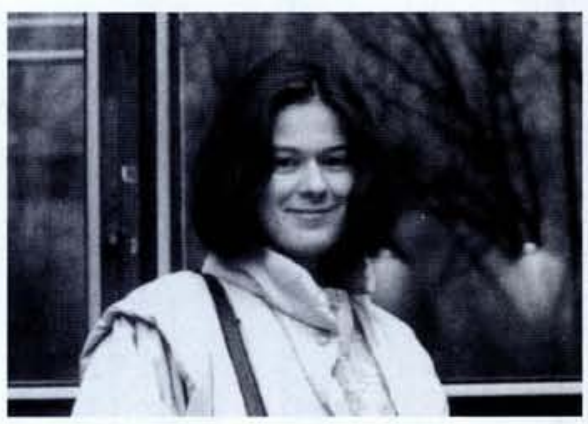
theoretical physics at the University of Geneva's Physics Department. In keeping with her secondary school's long tradition, she had learnt excellent French and was thus able to appreciate that things were "sympa" and well organized (the Department provided lodging in a hall of residence close by). She feels that while there is little difference between the levels of the courses in Geneva and back home, this is not the case for the overall programme (Katowice's is structured whereas Geneva's is flexible). The solution was to choose courses normally given in different years (she is taking 3rd and 4th year courses in statistical physics, field theory and relativity before returning home to write her 5 th year thesis on cosmology). Exams are naturally Katarzyna's main concern. The scheme is somewhat unique in that course credits are transferred so she must sit several exams over a short period in the summer; regular students, on the other hand, tend to have a more relaxed continuous assessment. She is confident, however, that her Coordinator will be able to help make the Department understand that she has this additional pressure.

from the regular budget (now about 950 MSFR p.a.). Discussion in Council would decide if modifications are needed to fit the resources allocated and any external contributions. The existing LEP machine would run until 2000, with LHC starting in 2002 after a 2 -year shutdown (the original proposals envisaged alternate operation).

CERN will to wait to see what US particle physicists want before considering cooperation. The US High-Energy Advisory Commit- tee's report on balancing short- and mediumterm ambitions after the SSC closure is due next June, so moves towards a LHC decision are pushed back to mid-1994, along with requests for technical proposals for detectors. Unknown is the reaction of Germany, CERN's largest contributor, which has argued that investments made in the 1980's should first be fully exploited. Meanwhile, the principle of equal access without at least charging user-related costs needs review.

\section{Why MathSoft Overseas has a centre of excellence for Mathematica at CERN}

\author{
Because Mathematica responds to CERN's needs for a comprehensively \\ supported mathematical software package for physics and engineering.
}

Mathematica represents a genuine revolution in the art of computing.

Collects in a single package available on any computer:

- Symbolics: Integrate [Exp[-x^2/2],x] $\rightarrow$ Sqrt[Pi/2]Erf[x/Sqrt[2]]

- Numerics: Erf[1.2] $\rightarrow 0.910314$

- Graphics: Plot[Erf[x],\{x,-4,4\}, AxesLabel $\rightarrow[$ "x","y"\}]

- List processing: Erf[\{1.1,2.5,-3.4\}] $\rightarrow\{0.7747609,0.9991862,-0.9999965\}$

- Functional programming with pattern recognition:

Factorial function $\rightarrow$ fac $\left[\mathrm{n} \_\right.$Integer/;n>0]: $=\mathrm{n}$ fac[n-1]; fac[0] $=1$

- The Descartes ${ }^{\mathrm{TM}}$ Library extends Mathematica

- Euclidian geometry: all Euclidian geometry on point, straight, circle, conics, lines, and polygons. Application to rigorous drawing, theorem testing, CAD, desktop publishing, and any graphics technique.

- Differential geometry of curves and surfaces.

- Visualization: pre-processing of objects to be visualized in the form of Cartesian surfaces, parametrric lines and surfaces, and geometric solids; data representation in needle tower or interpolating surfaces. Interface to AVS ${ }^{\mathrm{TM}}$.

- Integration of Mathematica by MathSoft Overseas in process control using UNIX

- Dedicated technical and commercial support

- Optimum pricing (volume discount, educational grants, site licensing, etc.).

- Three-day courses: Introduction to Mathematica, symbolic programming.

- Consulting on specific problems.

To become a centre of excellence in Mathematica, please contact:

MathSoft Overseas, CP 641, CH-1211 Geneva 3

Tel.: +41-22-346 52 60; Fax: +41-22-3465939

\section{Visualization of a parametric surface}

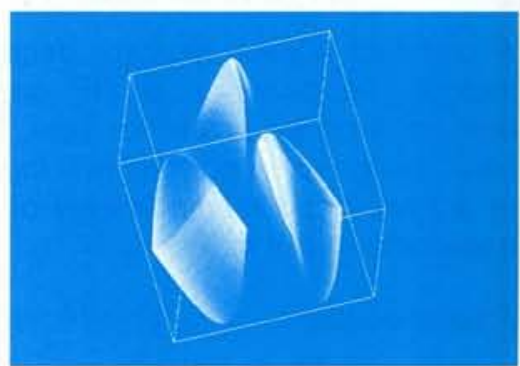

A figure produced with the statement: View $\left[\left\{\operatorname{Cos}[\mathrm{u}], \operatorname{Sin}[\mathrm{v}], \mathrm{u}^{*} \mathrm{v} / 10\right\},\{\mathrm{u},-\mathrm{Pi}, \mathrm{Pi}\},\{\mathrm{v},-\mathrm{Pi}, \mathrm{Pi}\}\right]$. Descartes Visualization acts as the link between Mathematica and the AVS ${ }^{\mathrm{TM}}$ visualization program. The rigorously defined object could, for example, represent stream flow. 